\title{
UHF-Band TV Transmitter for TV White Space Video Streaming Applications
}

\author{
Hyunchol Shin ${ }^{1, *} \cdot$ Hyukjun $\mathrm{Oh}^{2}$
}

\begin{abstract}
This paper presents a television (TV) transmitter for wireless video streaming applications in TV white space band. The TV transmitter is composed of a digital TV (DTV) signal generator and a UHF-band RF transmitter. Compared to a conventional high-IF heterodyne structure, the RF transmitter employs a zero-IF quadrature direct up-conversion architecture to minimize hardware overhead and complexity. The RF transmitter features I/Q mismatch compensation circuitry using 12-bit digital-to-analog converters to significantly improve LO and image suppressions. The DTV signal generator produces an 8-vestigial sideband (VSB) modulated digital baseband signal fully compliant with the Advanced Television System Committee (ATSC) DTV signal specifications. By employing the proposed TV transmitter and a commercial TV receiver, over-the-air, real-time, high-definition video streaming has been successfully demonstrated across all UHF-band TV channels between 14 and 69. This work shows that a portable hand-held TV transmitter can be a useful TVband device for wireless video streaming application in TV white space.
\end{abstract}

Key Words: DTV Signal Generator, RF Transmitter, TV Band Device, TV Transmitter, TV White Space.

\section{INTRODUCTION}

Television white space (TVWS) refers to a TV broadcasting spectrum that is geographically or temporarily unused by licensed users and at the same time can be shared by secondary users on an unlicensed basis. As specified by the Federal Communications Commission (FCC) regulatory rules $[1,2]$, the wide adoption of the TVWS devices in wireless local and regional area network services, such as IEEE 802.11af and IEEE 802.22, has been advocated for. Due to superior radio propagation characteristics at the ultra-high frequency (UHF) band, TVWS use scenarios include super-WiFi, long-range wireless sensor networks, disaster communication, entertainment multimedia links, wireless display links, among others. For example, industry-science-medical (ISM)-to-UHF-band RF converters $[3,4]$ is a TV-band device (TVBD) to enable Wi-Fi service in a TVWS band.

Another interesting usage model of TVBD is wireless video streaming. Since wireless video streaming technology has several attractive and useful applications, various approaches to realize the wireless video streaming have been reported in the literature. Specifically, an internet protocol-based MPEG (Moving Picture Experts Group) streaming technique operating in the wireless local area network was reported in [5], but it proved to be unstable and exhibit often interrupted and delayed performance. Millimeter-wave-band wireless display interface techniques, such as WirelessHD [6], IEEE 802.11ad [7], and ARIB STDB43 [8], can effectively provide high data throughput with low

Manuscript received January 7, 2019 ; Revised March 11, 2019 ; Accepted June 26, 2019. (ID No. 20190107-002J)

${ }^{1}$ Department of Electronics Convergence Engineering, Kwangwoon University, Seoul, Korea.

${ }^{2}$ Department of Electronics and Communications Engineering, Kwangwoon University, Seoul, Korea.

"Corresponding Author: Hyunchol Shin (e-mail: hshin@kw.ac.kr)

This is an Open-Access article distributed under the terms of the Creative Commons Attribution Non-Commercial License (http://creativecommons.org/licenses/by-nc/4.0) which permits unrestricted non-commercial use, distribution, and reproduction in any medium, provided the original work is properly cited.

(c) Copyright The Korean Institute of Electromagnetic Engineering and Science. 
latency but requires technically challenging developments of the millimeter-wave circuits and transceivers.

Another approach to wireless video streaming is employing a portable TV transmitter to send a standard TV broadcasting signal to a commercial TV receiver. It is worth noting that such a TV transmitter and its application to wireless video streaming appeared in an old article from the 1950s [9], in which a portable TV transmitter was developed and used as a military reconnaissance device. More recently, after the TVWS was officially approved, TVWS video streaming devices were also reported $[10,11]$ but lacked certain design and implementation details.

In this paper, we present a TV transmitter device that comprises a UHF-band RF transmitter and an Advanced Television System Committee (ATSC) digital TV (DTV) signal generator. The RF transmitter employs a zero-IF direct up-conversion architecture and features a I/Q mismatch calibration and power control for an improved signal-to-noise ratio. The ATSC modulator, primarily performing vestigial sideband (VSB) modulation and channel coding, is designed to be fully compliant with ATSC standards [12] in order to realize a seamless interface with commercially available TV receiver sets. By employing the proposed TV transmitter, we were able to successfully demonstrate wireless video streaming in all UHF TV channels.

\section{DESIGN}

Fig. 1 shows the TVWS wireless video streaming system architecture. Video and audio data are initially stored in a personal computer and fed to the ATSC modulator for VSB digital modulation. The modulated digital signal is fed to the RF transmitter for up-conversion to one of the UHF-band TV broadcasting channel frequencies. Finally, the RF signal is fed to an antenna and radiated through the air. On the receiver side, a commercial digital TV set receives the signal sent from the TV transmitter using the same antenna as the transmitter. With the TV channel properly set, the TV displays the received video and audio signals on the screen.

According to ATSC standards, the low-numbered TV channels between 2 and 13 reside within the VHF band (54-216 $\mathrm{MHz}$ ), and the high-numbered TV channels between 14 and 69 reside within the UHF band $(470-860 \mathrm{MHz})$. Among these, this work focuses on the UHF-band channels, because the VHF-band antenna is too large to be adopted for portable application. The UHF-band channels lie between 470 and 806 $\mathrm{MHz}$ and are divided into 6- $\mathrm{MHz}$ per each channel. For example, the first and last UHF-band channels are channel 14 (occupying 470-476 MHz) and channel 69 (occupying 800-806 $\mathrm{MHz})$, respectively. According to the TVWS specifications [1, 2], channels 14 to 20 are assigned to fixed TVBD, and channels 21 to 35 and 39 to 51 are assigned to fixed and mobile TVBD. Since the proposed TV transmitter of this work is to be used as either a fixed or mobile TVBD, it should support all UHFband channels between 14 and 69 .

Several UHF-band RF transmitters for TVWS application have been reported previously in the literature, most of which were based in heterodyne architecture and had high intermediate frequencies (IF) like $160 \mathrm{MHz}$ [13], $250 \mathrm{MHz}$ [14, 15], 280 $\mathrm{MHz}$ [16], and $488 \mathrm{MHz}$ [17]. Such high-IF frequencies would require high-Q IF filtering and a high sample-rate digital-to-analog converter (DAC) $[13,16,17]$ as well as significantly elevated hardware complexity $[14,15]$. In addition, these previous works did not incorporate ATSC-compliant DTV signal generators, so they could not be applied to a TV-band video streaming system. In contrast to previous high-IF heterodyne architectures, this work employs a zero-IF direct upconversion architecture.

Fig. 2 shows the block diagram of the RF transmitter. The digital baseband I/QDTV signal produced by the ATSC signal generator is fed to the DAC and converted into analog baseband I/Q signals. The 12-bit DAC ensures a 70-dB signal-tonoise ratio at the baseband analog output. The baseband analog signals are further filtered by the 6th-order Butterworth-type low-pass filters to minimize out-of-band noise and spurious components. The filter bandwidth is set to $6 \mathrm{MHz}$ to accommodate the TV channel bandwidth. A variable gain amplifier provides a gain tuning range between -20 and $+45 \mathrm{~dB}$ to improve the output dynamic range. The analog I/Q baseband signals are up-converted by the quadrature mixer and subsequently fed to a power amplifier to deliver an output power of up to $+20 \mathrm{dBm}$ before finally being radiated through a UHF-band antenna.

The quadrature balanced-type mixer topology produces a sin-
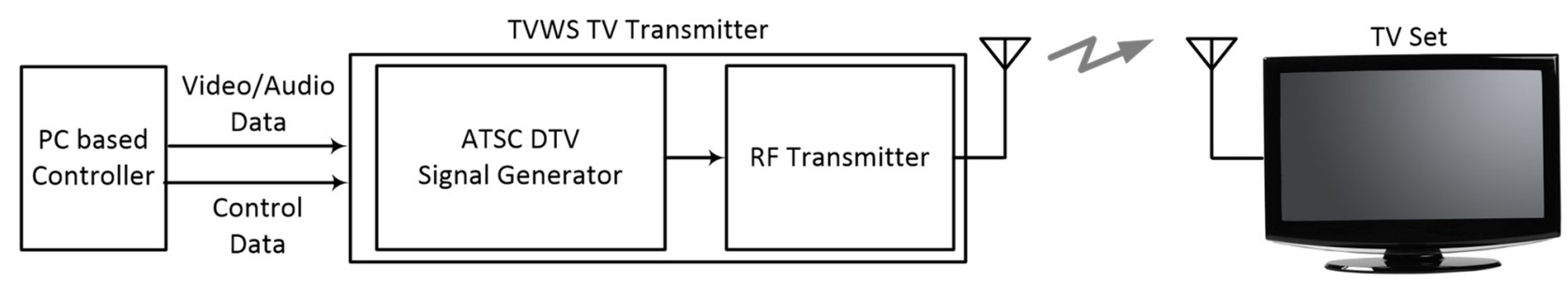

Fig. 1. TV white space band wireless video streaming system. 


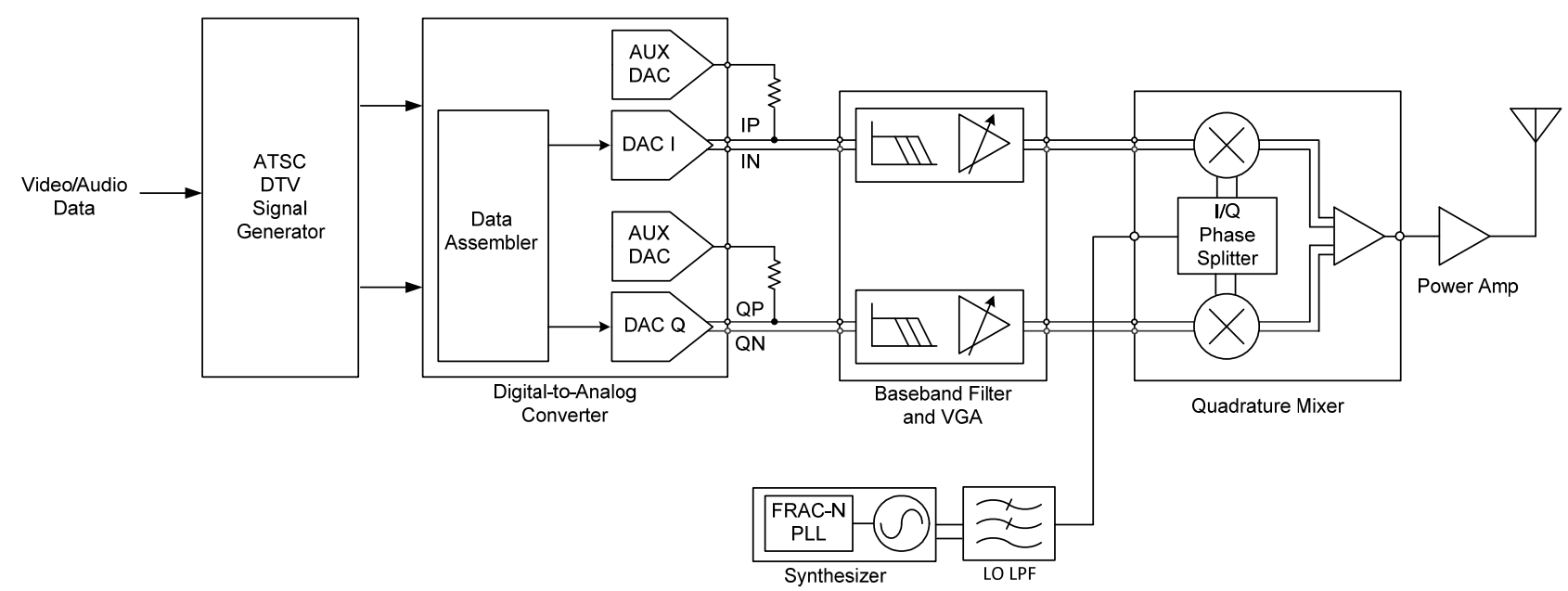

Fig. 2. Block diagram of UHF-band TV transmitter.

gle side-band mixing product with suppressed local oscillator (LO) and image components. Since the residual LO and image leakage components degrade output signal purity, the I/Q mismatch and dc offset must be properly calibrated. In this design, the I/Q amplitude mismatch was compensated for by controlling the DAC's output dynamic range, while the dc offsets of the I/Q differential signals were compensated for by controlling the two auxiliary and main DAC's output common mode levels. Such compensation circuitry significantly suppresses the LO and image leakage components at the output signal.

Theoretical analysis on LO and image suppression levels was carried out to determine the minimum required DAC resolution. Assuming the I/Q baseband signals are given by:

$$
\begin{gathered}
V_{B B, I}=V_{o}(1+\varepsilon) \cos \left(\omega_{B B} t+\Delta \theta\right)+V_{o s} \\
V_{B B, Q}=V_{o} \sin \left(\omega_{B B} t\right)
\end{gathered}
$$

where $V_{o}$ is the amplitude, $\omega_{B B}$ the baseband signal frequency, $\varepsilon$ the amplitude error, $\Delta \theta$ the phase error, and $V_{o s}$ the dc offset. When (1) and (2) are multiplied and combined with $\cos \left(\omega_{c} t\right)$ and $\sin \left(\omega_{c} t\right)$, respectively, in which $\omega_{c}$ is the RF carrier frequen$\mathrm{cy}$, the resulting output signal can be decomposed into three components of the lower-side band signal $V_{L S B}$, the uppersideband signal $V_{U S B}$, and the LO component $V_{L O}$. They are written as the following:

$$
\begin{aligned}
V_{L S B}= & \frac{V_{o}}{2}[(1+\varepsilon) \cos \Delta \theta-1] \cdot \cos \left(\left(\omega_{c}-\omega_{B B}\right) t\right) \\
& +\frac{V_{o}}{2}(1+\varepsilon) \sin \Delta \theta \cdot \sin \left(\left(\omega_{c}-\omega_{B B}\right) t\right) \\
V_{U S B}= & \frac{V_{o}}{2}[(1+\varepsilon) \cos \Delta \theta+1] \cdot \cos \left(\left(\omega_{c}+\omega_{B B}\right) t\right) \\
& -\frac{V_{o}}{2}(1+\varepsilon) \sin \Delta \theta \cdot \sin \left(\left(\omega_{c}+\omega_{B B}\right) t\right)
\end{aligned}
$$

$$
V_{L O}=V_{o s} \cos \left(\omega_{c} t\right)
$$

Assuming (3) is the wanted tone, the image rejection ratio (IRR) and LO rejection ratio (LOR) are given as the following:

$$
\begin{gathered}
I R R=\frac{(1+\varepsilon)^{2}-2(1+\varepsilon) \cos \Delta \theta+1}{(1+\varepsilon)^{2}+2(1+\varepsilon) \cos \Delta \theta+1} \\
L O R=\left(\frac{2 \cdot V_{o s}}{V_{o}}\right)^{2}
\end{gathered}
$$

From (6) with an assumption of zero phase mismatch $\Delta \theta=0$, the amplitude mismatch $\varepsilon$ must be less than $1.98 \times 10^{-2}$ for 40 $\mathrm{dB}$ IRR. This indicates that at least a 6-bit resolution is needed for the DAC's amplitude control. On the other hand, from (7), the dc offset $V_{o s}$ must be less than 1/200 of the baseband amplitude for $40 \mathrm{~dB}$ LOR. This indicates that at least an 8-bit resolution is needed for the DAC's dc level control. In the present implementation, the 12-bit resolution DAC was employed in order to sufficiently meet the image and LO rejection requirements.

The fractional-N phase-locked loop (PLL) synthesizer properly sets the LO frequency according to the wanted channel number. The low-pass filter placed at the PLL output is used to filter out the residual spurious and harmonic tones at the LO signal. The single-phase LO signal is converted to I/Q LO signals by the phase splitter placed in front of the mixers.

Commercially available off-the-shelf parts were used to realize the RF transmitter in Fig. 2. The DTV signal generator was implemented in a field-programmable gate array chip of Xilinx Kintex 7 (Xilinx, San Jose, CA, USA). A low-power broadband mixed-signal front-end chip, AD9963, from Analog Device Inc. (Norwood, MA, USA) converted the serial digital bit stream into a digital I/Q signal using an on-chip data assembler and subsequently converted the digital I/Q signal into differential 
analog I/Q signals using two 12-bit DACs. The auxiliary DACs for $\mathrm{I} / \mathrm{Q}$ mismatch compensation were also available from the same chip. The baseband analog processor performing the LPF and VGA functions, the up-conversion quadrature mixer with on-chip quadrature LO generator, and the fractional-N PLL synthesizer were all realized by employing ADRF6516, ADL5375, and ADF4351, respectively, all of which were from Analog Device Inc. Additionally, the power amplifier chip, TQP3M9009, was from TriQuint Semiconductor Inc., Hillsboro, OR, USA. For the three signal-path chips of ADRF6516, ADL5375, and ADF4351, the power gains were -20 to +40 , 3 , and $+26 \mathrm{~dB}$, while their $1-\mathrm{dB}$ compression powers were +3 , +9.6 , and $+20.7 \mathrm{dBm}$, respectively. Thus, the entire transmitter provided a typical output power of $0 \mathrm{dBm}$, while its peak output power reached up to $+20 \mathrm{dBm}$. Two DTV antennas, LP49 from Spectrum Inc., Seoul, Korea with $6 \mathrm{dBi}$ gain were used for the transmitter and receiver antennas. Meanwhile, it is also worth noting that if a single-chip CMOS RF transmitter integrated circuit, such as the one featured in the author's previous works [18], can be adopted, the whole TV transmitter can be realized with lower power consumption and a smaller form factor.

Fig. 3 shows the block diagram of the DTV signal generator based on the ATSC DTV specifications. It was designed to be compliant with the ATSC standards [12, 19]. First, the input MPEG transport stream (MPEG-TS) data packet was randomized to transform the bit sequences into quasi-pseudorandom binary sequences. The subsequent Reed-Solomon encoder added a 20-byte forward error correction (FEC) code to the 188-byte input packet to protect it from erroneous reception. The data interleaver enhanced immunity to burst-type noise and interference, while the trellis coded modulation (TCM) with a two-thirds rate further improved the data demodulation performance. A 4-state optimal Ungerboeck code was used for TCM encoding. Then, the segment and field sync signals were multiplexed with the trellis encoded data in order to help the receiver acquire data segment and data field synchronizations. An additional assistant pilot signal was added to provide a convenient method for measuring the frequency of the VSB signal at the receiver end. Finally, 8-VSB modulation was carried out to produce the wanted DTV digital baseband signal. The total
ATSC signal generator was implemented in a field programmable gate array (FPGA), and the FPGA board was then interfaced with the RF transmitter and the personal computer controller, as illustrated in Fig. 1.

\section{RESULTS}

The fabricated TV transmitter is shown in Fig. 4(a). It comprises the RF transmitter board and the DTV signal generator FPGA board. Fig. 4(b) shows the RF transmitter module in more detail and has each circuit block denoted. A four-layer FR-4 substrate was used for the RF board, for which the top and bottom layers were used for signal routing, and the inner two layers were used for ground and supply planes.

The performance of the TV transmitter was tested by examining the output spectrum. The analog baseband DTV signal

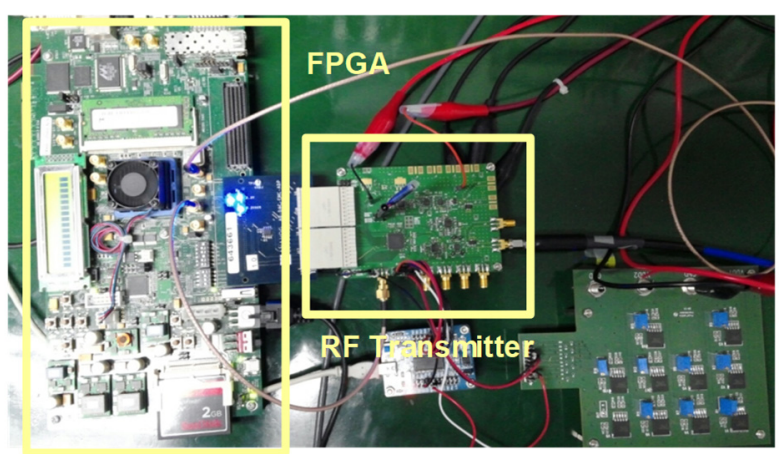

(a)

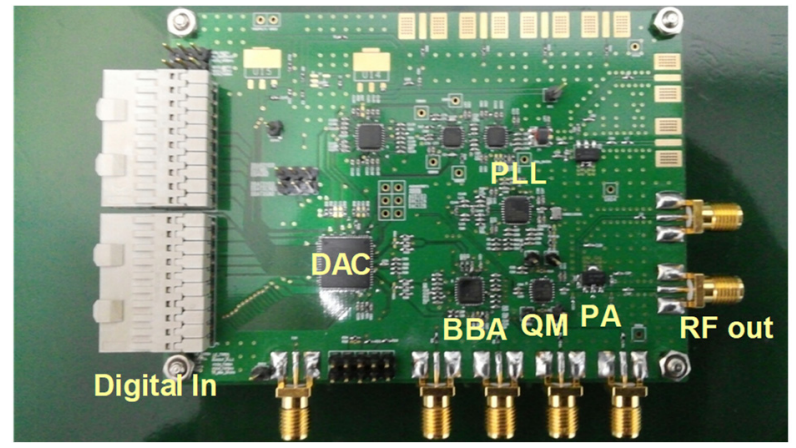

(b)

Fig. 4. (a) Photograph of TV transmitter comprising DTV signal generator FPGA board and RF transmitter board. (b) Photograph of RF transmitter board.

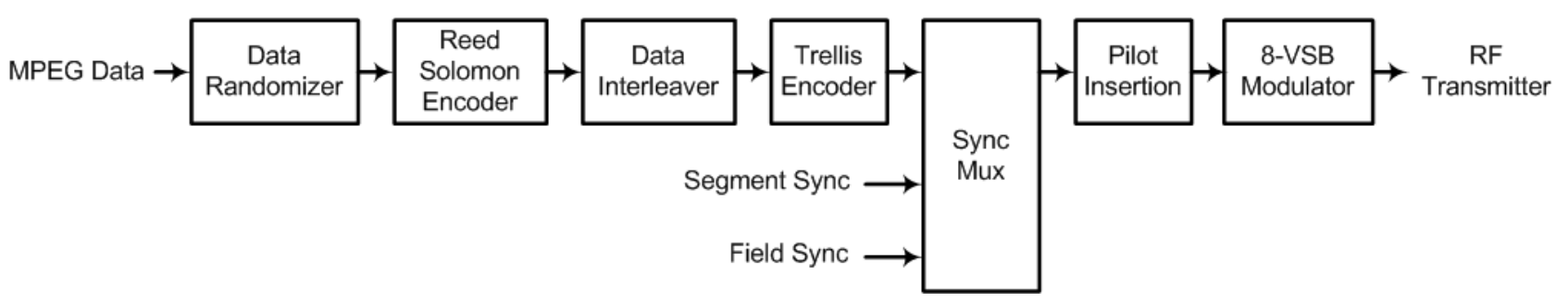

Fig. 3. DTV signal generator. 
spectrum measured at the DAC output is shown in Fig. 5. Since the DTV signal was VSB-modulated with a $6 \mathrm{MHz}$ bandwidth, the signal spectrum appeared in 0 to $+6 \mathrm{MHz}$, while the image signal simultaneously appeared in 0 to $-6 \mathrm{MHz}$. According to the ATSC DTV specifications, the total bandwidth of $6 \mathrm{MHz}$ can be broken down into a half-power bandwidth of $5.38 \mathrm{MHz}$ and both-side steep transitions of a 310 $\mathrm{kHz}$ width. Additionally, a pilot signal appeared at 309.441 $\mathrm{kHz}$ away from the band low end. These characteristics were well verified through the baseband spectrum.

Fig. 6 is the measured RF output spectrum when a 3-MHz single-tone baseband signal and $473-\mathrm{MHz} \mathrm{LO}$ signal were applied. As can be seen, the wanted RF tone appeared at 470 $\mathrm{MHz}$, as the quadrature mixer performed lower single-sideband mixing. Note that $\mathrm{LO}$ and image leakage suppression when the output power was $-18.5 \mathrm{dBm}$ was 32 and $43 \mathrm{dBc}$ at $473 \mathrm{MHz}$ and $476 \mathrm{MHz}$, respectively.

LO and image leakage powers and suppression levels were

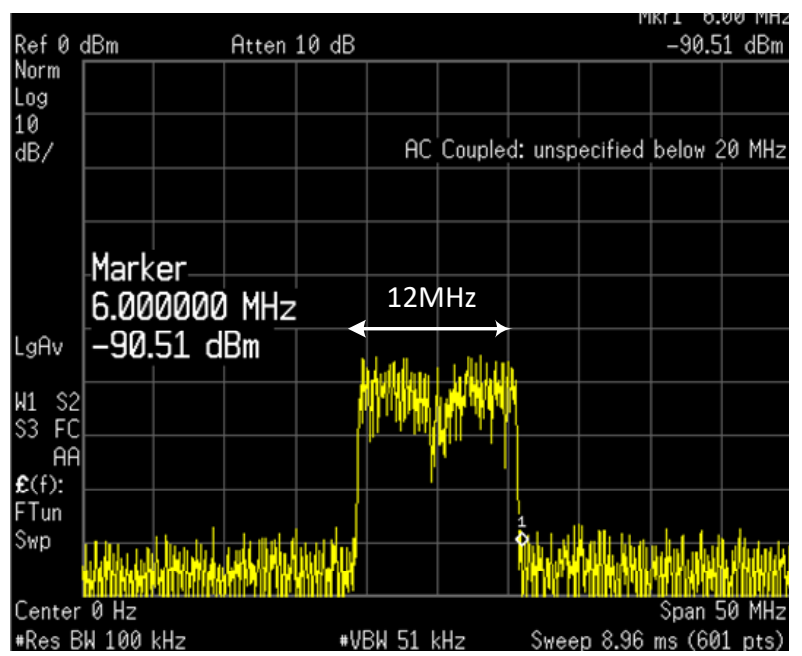

Fig. 5. Measured spectrum of the analog baseband DTV signal.

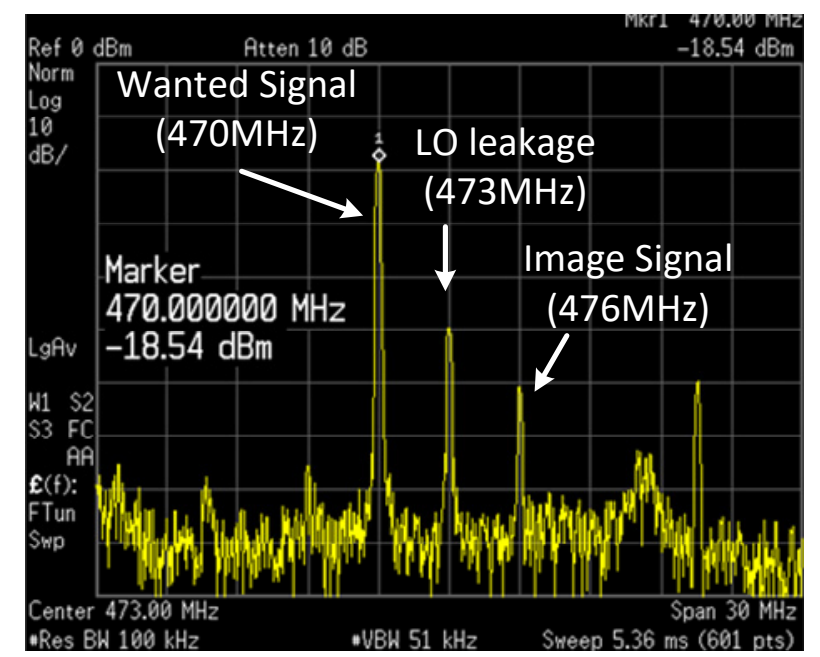

Fig. 6. Measured RF spectrum with a 3-MHz single-tone baseband and 473-MHz LO signals applied. further characterized across output power levels from -33 to $+22 \mathrm{dBm}$ for five TV channels: $14,27,41,55$, and 69. The measured results are illustrated in Fig. 7. The LO leakage power level was -40 to $-52 \mathrm{dBm}$, and the LO suppression was about +6 to $+75 \mathrm{dBc}$. It should be noted that the LO leakage power level did not vary significantly across output power levels. Hence, the LO suppression levels improved as the output power increased. This behavior can be explained by Eq. (7) when recognizing that the dc offset does not greatly vary but remains almost constant while the output signal level increases. On the other hand, the image leakage power level was about -21 to -76 $\mathrm{dBm}$, and the corresponding image suppression level was about 35 to $62 \mathrm{dBc}$. It should also be noted that the image leakage power tended to increase as the output power increased. This behavior can be understood through Eq. (6) by recognizing that the amplitude mismatch e tends to remain almost constant as the output power level increases.

The entire TV transmitter was tested for over-the-air wireless link with a commercial DTV set used as a receiver. Fig. 8 shows the test setup. Test video data that was stored in a personal computer was transformed into the DTV signal using the DTV

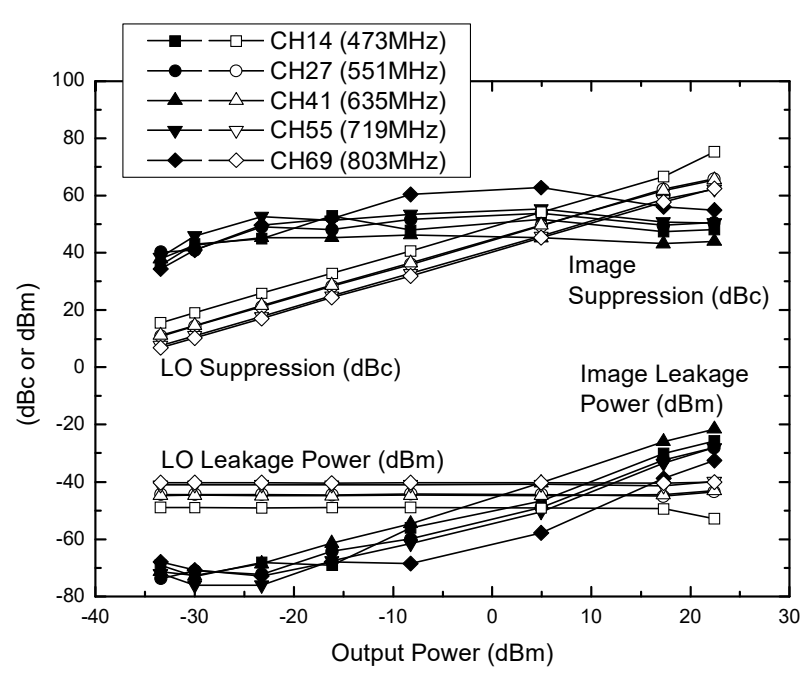

Fig. 7. LO and image leakage power and suppression level across five TV channels in respect to output power.

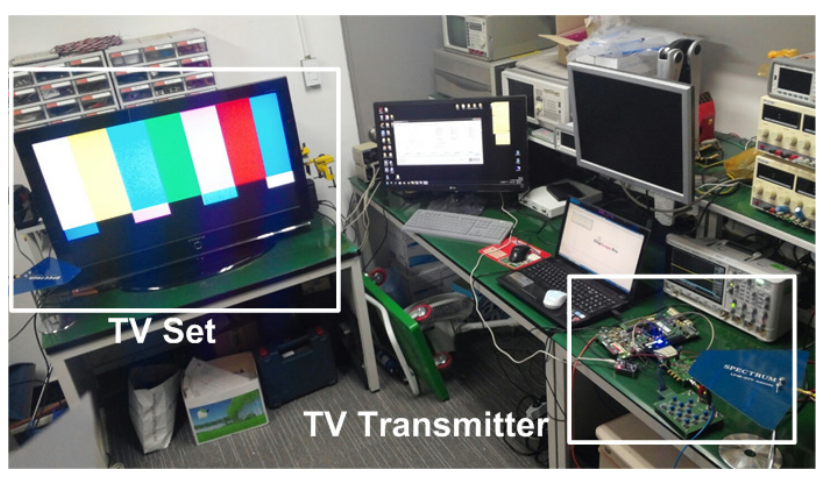

Fig. 8. Over-the-air test. 
signal generator and up-converted by the RF transmitter to one of the selected TV channels between 14 and 69 before being finally sent out over the air via an antenna. A commercial DTV receiver was placed two meters away from the transmitter, which used the same antenna as the transmitter.

Fig. 9 displays the received images of several selected TV channels. As can be seen, test images were successfully transmitted and received for channels $14,15,37$, and 69 . Note that the TV screen automatically displayed the channel number in the upper left corner, as can be seen in Fig. 9. A color bar test image was displayed for channels $14,37,69$, and a cartoon video was displayed for channel 15 .

The performance of the fabricated TV transmitter is summarized in Table 1. It should be noted that the previous TVWS devices in $[3,4]$ did not comprise a full RF transmitter but only a Wi-Fi-to-UHF frequency converter. Also, note that the previous TV-band transmitters in [13-17] only demonstrated UHF-band RF transmitters without ATSC DTV signal generator. In contrast, this work fully realized the UHF-band RF transmitter and ATSC DTV signal generator while successfully demonstrating TV signal wireless streaming to a commercial DTV set. Overall, the experimental results successfully show that the proposed TV transmitter is instrumental as a wireless video streaming device, exhibiting the possibility that a portable

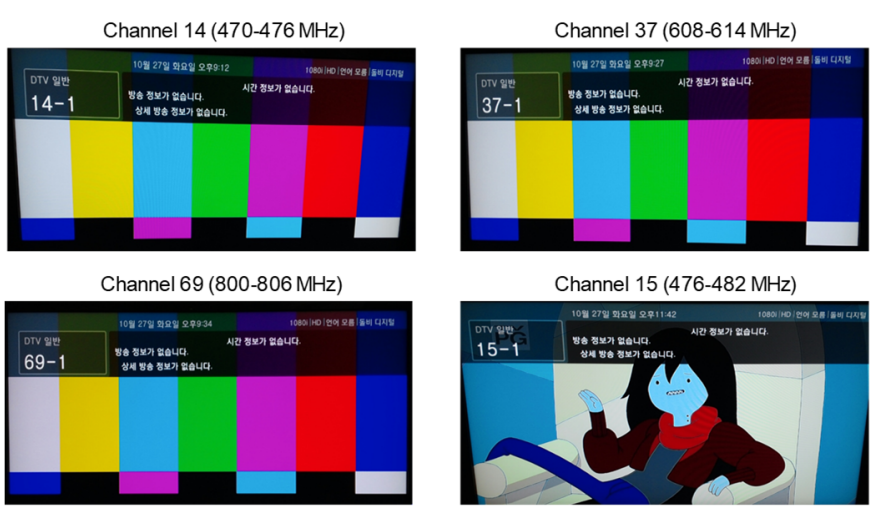

Fig. 9. Received test images for TV channels 14, 15, 37, and 69.

Table 1. Performance summary

\begin{tabular}{lc}
\hline \multicolumn{1}{c}{ Parameter } & Measured performance \\
\hline Frequency band $(\mathrm{MHz})$ & $470-806$ \\
Supported TV channels & $14-69$ \\
Supported video resolution & HD 1080i $(1920 \times 1080)$ \\
Digital modulation & ATSC-compliant 8-VSB \\
Signal path gain $(\mathrm{dB})$ & $0-65$ \\
Output-referred $1 \mathrm{~dB}$ compression & +20 \\
$\quad$ power $(\mathrm{dBm})$ & 0 \\
Typical output power $(\mathrm{dBm})$ & 3.3 and 5 \\
Supply voltage $(\mathrm{V})$ & \\
\hline
\end{tabular}

TV transmitter can be a useful TVBD for wireless video streaming application in TVWS.

\section{CONCLUSION}

A TV transmitter composed of a DTV signal generator and a UHF-band RF transmitter was developed for wireless video streaming application in TVWS. The zero-IF direct conversion RF transmitter supported UHF-band TV channels 14 to 69 (470-860 MHz). LO and image leakage calibration was implemented using 12-bit DACs to achieve significant improvements in LO and image suppression. The DTV signal generator implemented in FPGA produced a DTV digital signal fully compliant with the ATSC specifications. The experimental results successfully demonstrated that over-the-air wireless video streaming is possible using the proposed TV transmitter module as a transmitter and a commercial TV set as a receiver. In conclusion, this work reveals that a portable hand-held TV transmitter can be a useful TVBD for TVWS application.

This work was supported by the Institute for Information and Communications Technology Promotion (IITP) grant funded by the Korean Government (MSIT) (No. 2017-000959, University Basic IT Research Center).

\section{REFERENCES}

[1] Federal Communications Commission, "Second report and order and memorandum opinion and order," FCC 08-260, 2008.

[2] Federal Communications Commission, "Third memorandum opinion and order: TVBD spectrum requirements," FCC 12-36, 2012.

[3] A. Ashok, I. Subbiah, G. Varga, M. Schrey, and S. Heinen, "Radio front-end enabling WLAN over white space cognitively," in Proceedings of IEEE 15th Annual Wireless and Microwave Technology Conference, Tampa, FL, 2014, pp. 1-5.

[4] T. Matsumura and H. Harada, "Prototype of tablet-type TV band portable device with UHF converter for TV white-spaces utilization," in Proceedings of IEEE 24th International Symposium on Personal, Indoor and Mobile Radio Communications, London, UK, 2013, pp. 2748-2752.

[5] T. Shida, T. Sato, H. Nakayama, H. Kosaka, and K. Sugiyama, "Robust HD video stream transmission for wireless DTV," IEEE Transactions on Consumer Electronics, vol. 53, no. 1, pp. 96-99, 2007.

[6] J. M. Gilbert, C. H. Doan, S. Emami, and C. B. Shung, "A 4-Gbps uncompressed wireless HD A/V transceiver chipset," IEEE Micro, vol. 28, no. 2, pp. 56-64, 2008.

[7] C. Cordeiro, "The pursuit of tens of gigabits per second 
wireless systems," IEEE Wireless Communications, vol. 20, no. 1, pp. 3-5, 2013.

[8] S. Suzuki, T. Nakagawa, and T. Ikeda, "Development of millimeter-wave mobile camera and performance improvement in outdoor LOS environment," IEICE Transactions on Fundamentals of Electronics, Communications and Computer Sciences, vol. 93, no. 11, pp. 2099-2107, 2010.

[9] No author, "Portable television transmitter is used in field reconnaissance work," Electrical Engineering, vol. 75, no. 5, pp. 482-483, 1956.

[10] V. R. Raveendran, Y. A. Wang, J. H. Choi, and C. T. Nguyen, "Controlling multimedia device in remote display mode," US Patent Publications 20110219420A1, September 8, 2011.

[11] S. W. Oh, Y. Zeng, W. Zhang, S. Naveen, and F. Chin, "TV white-space video streaming demo," in Proceedings of IEEE Symposium on New Frontiers in Dynamic Spectrum (DySPAN), Singapore, 2010.

[12] W. Bretl, W. R. Mentel, G. Sgrignoli, X. Wang, S. M. Weiss, and K. Salehian, "ATSC RF, modulation, and transmission," Proceedings of the IEEE, vol. 94, no. 1, pp. 44-59, 2006.

[13] K. M. Kang, J. C. Park, and S. Park, "Implementation of filter bank-based RF transceiver for TV white space," ETRIJournal, vol. 37, no. 6, pp. 1077-1086, 2015.

\section{Hyunchol Shin}

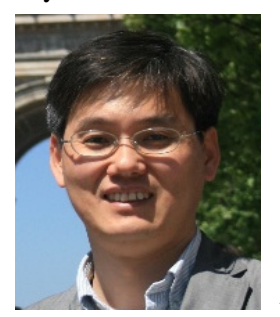

received his B.S., M.S., and Ph.D. degrees in electrical engineering from the Korea Advanced Institute of Science and Technology (KAIST), Daejeon, Korea, in 1991, 1993, and 1998, respectively. During his Ph.D., he held an internship as a doctoral student in 1997 at the Daimler-Benz Research Center, Ulm, Germany. Upon completion of his Ph.D., he worked at several research institutions and companies, including Samsung Electronics, Suwon, Korea, University of California at Los Angeles, CA., USA, and Qualcomm, San Diego, CA, USA, where he had been involved in RF/analog/microwave circuit design for wireless communications. Since 2003, he has been with Kwangwoon University, Seoul, Korea, where he is currently a professor with the Department of Electronics Convergence Engineering. In 2010-2011, he took his sabbatical leave with Qualcomm, San Diego, CA, USA. He is currently the Director of the Radio Research Center for Advanced mm-Wave Beamforming Technology. He has co-authored over 80 journal and conference papers and holds over 30 patents in the field of RF/analog circuit design. His research interests focus on CMOS RF/analog/millimeter-wave circuits and beamforming antenna systems for $5 \mathrm{G}, 60 \mathrm{GHz}$, and IoT applications. Prof. Shin has served on Technical Program Committees of several IEEE conferences, such as the International Solid-State Circuits Conference (ISSCC) from 2015-2018, the VLSI Circuit Symposium (VLSI) from 2016-2018, the Asian Solid-State Circuit Conference (A-SSCC) from 2007-2012, and the Midwest Symposium on Circuits and Systems (MWCAS) in 2011. He also served as the Technical Program Committee Chair and General Chair for the International System-on-Chip Design Conference (ISOCC) in 2017 and 2019, respectively.
[14] C. You, X. Zhu, X. Zhang, J. Liu, Z. Cao, J. Chen, L. N. Quyen, and W. Zong, "Study of RF subsystem used in dynamic spectrum sharing system at TV band," IEEE Transactions on Industrial Electronics, vol. 60, no. 6, pp. 23462357, 2013.

[15] C. You, X. Zhu, J. Liu, X. Zhang, J. Chen, and Z. Cao, " Design of RF subsystem for dynamic spectrum sharing system in UHF band," Microwave and Optical Technology Letters, vol. 53, no. 9, pp. 2093-2100, 2011.

[16] M. Schuhler, A. Jaschke, M. Tessenma, and C. Kelm, " Flexible RF front-end for communication in TV white spaces," in Proceedings of the 43rd European Microwave Conference, Nuremberg, Germany, 2013, pp. 1087-1090.

[17] D. McCloskey and P. Gossett, "Wideband transceiver architecture for TV whitespace applications," in Proceedings of IEEE Symposium on New Frontiers in Dynamic Spectrum (DySPAN), Singapore, 2010, pp. 1-7.

[18] S. Kim, J. Sohn, and H. Shin, "A CMOS UHF harmonic rejection transceiver with 2-D LO phase calibration for TV white space applications," IEEE Transactions on Circuits and Systems II: Express Briefs, vol. 64, no. 11, pp. 12971301, 2017.

[19] S. W. Heo and H. Kim, "Multi-channel DTV signal generator design using modified farrow filter," IEICE Electronics Express, vol. 23, no. 8, pp. 1954-1960, 2011.

Hyukjun Oh

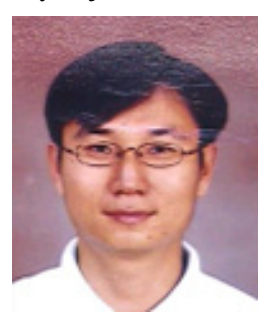

received his B.S., M.S., and Ph.D. degrees from the Korea Advanced Institute of Science and Technology (KAIST), in 1993, 1995, and 1999, respectively. From 1999 to 2000, he was with the Department of Electrical Engineering at Stanford University, where he performed research on next generation wireless communication systems based on CDMA and OFDM technologies. From 2001 to 2004, he was with Qualcomm Inc., USA, where he worked on developments of MSM 6000 series for 3GPP UMTS. Since 2004, he has been with the Department of Electronics and Communications Engineering at Kwangwoon University, Seoul, Korea, where he is currently a professor. His research interests include signal processing algorithms for future cellular networks as well as modem designs for wireless communications. 